\title{
Simulating Two-Dimensional Stick-Slip Motion of a Rigid Body using a New Friction Model
}

\author{
Yunsu Na $^{1}$, Sherif El-Tawil ${ }^{1}$, Ahmed Ibrahim², Ahmed Eltawil ${ }^{2}$ \\ ${ }^{1}$ University of Michigan, 2374 G.G. Brown, Ann Arbor, Michigan, USA. \\ yunsu@umich.edu; eltawil@umich.edu \\ ${ }^{2}$ University of California, Irvine, 4412 Engineering Hall, Irvine, California, USA. \\ amibrah1@uci.edu; aeltawil@uci.edu
}

\begin{abstract}
This paper proposes a new method for simulating the two dimensional stick-slip response of a rigid body subjected to friction forces. The physical problem is complicated by the fact that the two-dimensional equations of motion are coupled and that the two separate directional responses are highly nonlinear, particularly at transition points, when the relative velocity between the rigid body and supporting surface is close to zero. The proposed model does not employ the commonly used imaginary spring concept, which is numerically convenient, but has no physical meaning. Rather, it extends Westermo and Udwadia's one-dimensional friction method to two dimensions. The proposed model explicitly solves the governing equation of motion by employing an interpolation technique and numerical integration to find exact transition points in the velocity response curve. Using simulation, the new model is compared to existing models for cases involving harmonic motions and is shown to overcome certain numerical problems associated with existing models.
\end{abstract}

Keywords: Friction model, rigid body, stick-slip

\section{Introduction}

This paper is motivated by the considerable interest in the earthquake engineering community to detect occurrences of earthquakes and acquire acceleration data from smart devices in buildings affected by an earthquake, without installation of dedicated sensors. The premise is that smart devices located in the buildings can be used to compute floor displacements from the recorded accelerations, which can then enable automated damage assessment methods. Therefore, a deeper understanding must be developed of the motion of non-fixed rigid bodies (in this case, smart devices) on flat surfaces in a seismically excited building. With this as motivation, the general objective of this paper is to develop a new model to represent the contact kinematics between a rigid body and an underlying surface.

A popular friction model proposed by Griffin [1] uses an imaginary spring between two contact surfaces to model one-dimensional tangential relative displacement under constant normal load. However, no attempt was made to relate the stiffness of the spring to physical values. Spring stiffness is empirically selected in practice. Using a different approach, Westermo and Udwadia [2] developed a friction model to represent a simplified rigid block that is supported on a rigid surface without utilization of an imaginary spring. The acceleration of the block is calculated based on transition criteria of stick and slip motion. The Westermo and Udwadia [2] model addresses one-dimensional motion and cannot be directly applied to two dimensions, where the frictional effects can be highly non-linear, particularly when the friction force is distributed to each principal direction. Furthermore, there is the additional complication that the equations of motions are coupled in two dimensions.

In this paper, a friction model is proposed based on the ideas of Westermo and Udwadia [2]. The proposed model extends the original formulation to two dimensions. The new model is validated by comparing its response to the commonly used Griffin [1] model.

\section{Frictional Model and Equation of Motion}

Consider a rigid block sitting on a surface in one dimension as shown in Fig 1. The surface is subjected to a certain motion such as harmonic motion or earthquakes described by acceleration of surface, $\ddot{w}(t)$. The non-fixed block on the surface can undergo stick-slip motion and the equation of motion in the $x$-direction is defined as 


$$
m \ddot{u}(t)=F_{f}
$$

where $m$ is the mass of the block, $\ddot{u}(t)$ is acceleration of block, and $F_{f}$ is the friction force acting between surface and block.

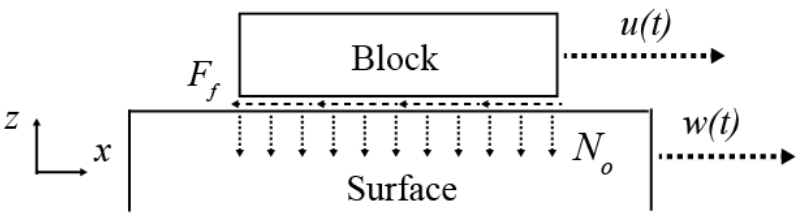

(a) Westermo and Udwadia's friction model

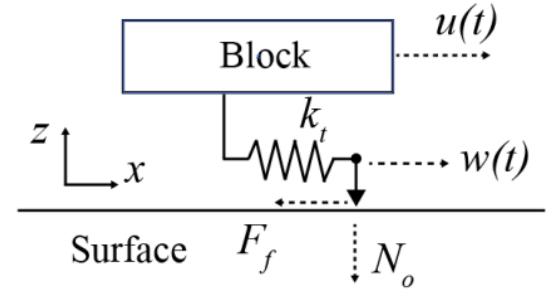

(b) Griffin's friction model

Fig 1. Schematic diagram of a block on an underlying surface.

\subsection{Westermo and Udwadia's One Dimensional Friction Model}

Westermo and Udwadia [2] presented a one dimensional stick-slip model as shown in Fig 1a. In stick mode, the velocity of the surface and block are equal $(\dot{u}(t)=\dot{w}(t))$, which means that the relative velocity of the block with respect to the surface is zero. The block remains in stick mode until the resultant of the inertia force mü exceeds the friction force. During sliding, the Coulomb kinetic friction force is used with a constant coefficient of static and dynamic friction in value, $\mu$. For simplicity the normal load is a constant value, $N_{o}=m g$, where $m$ is the mass of the block and $g$ is the acceleration due to gravity. Under such conditions, sliding of the block initiates and motion is controlled by the equation obtained from equilibrium of the forces acting on the block, as shown in Eq. (2). The sign of the inertial force is the same as the sign of the surface acceleration. After sliding, velocity is calculated as a function of the time of initiation of sliding $t_{i}$ in Eq. (3). The sliding mode ends when the velocities of the block and surface are the same $(\dot{u}(t)=\dot{w}(t))$.

$$
\begin{gathered}
|m \ddot{u}(t)|=\mu g m \\
\dot{u}(t)=\operatorname{sign}\left(\ddot{w}\left(t_{i}\right)\right) \mu g\left(t-t_{i}\right)+\dot{u}\left(t_{i}\right)
\end{gathered}
$$

\subsection{Griffin's [1] Spring Mass Friction Model (SMFM)}

According to Griffin [1], motion occurs in stick or slip modes. In the former, the tangential friction force is calculated by multiplying the relative displacement by the imaginary spring's stiffness, $k_{t}$ as shown in Fig $1 \mathrm{~b}$. In this case, the imaginary spring is in the elastic range in Eq. (4). In the latter, the friction force is equal to the Coulomb limit $\left(\mu N_{o}\right)$ such that the friction is no longer represented by an elastic stiffness relationship. The sign of the friction force is determined by the direction of slipping. For two-dimensional motion, Griffin and Menq suggested a model with two orthogonal springs [3] and [4] and assumed an isotropic coefficient of friction between the contact surfaces. In slipping mode, the tangential friction forces, $F_{f x}$ and $F_{f y}$ in $x$ and $y$ direction, respectively, are determined by the instantaneous sliding velocity unit vector, which consequently results in coupling of the $x$ and $y$ equations of motion in Eq. (6). The relative velocities in both direction between

the block and the surface are defined as $\dot{X}(t)=\dot{u}_{x}(t)-\dot{w}_{x}(t)$ and $\dot{Y}(t)=\dot{u}_{y}(t)-\dot{w}_{y}(t)$, where $\dot{u}_{x}(t), \dot{u}_{y}(t), \dot{w}_{x}(t)$ and $\dot{w}_{y}(t)$ are the velocity of the block and surface in two dimensions, respectively.

$$
\begin{gathered}
F_{f}=k_{t}(u(t)-w(t))=k_{t} X(t) \\
F_{f}=\operatorname{sign}\left(\dot{w}\left(t_{i}\right)\right) \mu N_{o} \\
{\left[\begin{array}{c}
F_{f_{x}} \\
F_{f_{y}}
\end{array}\right]=\frac{\mu N_{o}}{\sqrt{(\dot{X}(t))^{2}+(\dot{Y}(t))^{2}}}\left[\begin{array}{c}
\operatorname{sign}\left(\dot{w}_{x}\left(t_{i}\right)\right) \dot{X}(t) \\
\operatorname{sign}\left(\dot{w}_{y}\left(t_{i}\right)\right) \dot{Y}(t)
\end{array}\right]}
\end{gathered}
$$




\subsection{Proposed Two Dimensional Friction Model}

Two problems that mar previous models are: 1) a constant time step is used for numerical simulation, which makes it difficult to find an accurate transition point when the direction of relative velocity changes; 2 ) the friction force is distributed in $x$ and $y$ directions as a function of the magnitude of relative velocities in two directions. However, this criterion leads to unreasonable force distributions at the transition points, particularly in situations where only one relative velocity is changing the direction while the other is not.

To address the first point, an interpolation technique is applied to more accurately compute transition points. At transition points, the time step is discretized into multiple sub-steps using a cubic spline. Experimentation with various numbers of steps showed that 20 sub-steps produces reasonable answers. To address the second problem, a new criterion is proposed to distribute the friction force using a modification of Tariku and Roger's assumption [5]. The original model proposed by Tariku and Roger [5] assumes that a block accelerates in the direction of the inertial force regardless of its previous velocity direction in the micro-slip region. They considered the micro-slip region to be that where the magnitude of the sliding velocity is less than $0.1 \mathrm{~mm} / \mathrm{s}$ [5]. In this work, the Tariku and Roger assumption is modified as follows: 1) CASE 1, where relative velocity is changing in only one direction, the accelerations of the block are calculated as a function of the magnitude of the accelerations of the surface in each direction, see Eq. (7). 2) CASE 2, when the relative velocities are changing in two directions, the accelerations of the block are calculated as outlined by Eq. (6). The difficulty with Eqs. (6) and (7) is that they represent a set of coupled differential equations. Both sets are solved numerically using the Runge Kutta method.

$$
\left[\begin{array}{l}
\ddot{u}_{x}(t) \\
\ddot{u}_{y}(t)
\end{array}\right]=\frac{\mu g}{\sqrt{\ddot{w}_{x}(t)^{2}+\ddot{w}_{y}(t)^{2}}}\left[\begin{array}{c}
\operatorname{sign}\left(\dot{w}_{x}\left(t_{i}\right)\right) \ddot{w}_{x}(t) \\
\operatorname{sign}\left(\dot{w}_{y}\left(t_{i}\right)\right) \ddot{w}_{y}(t)
\end{array}\right]
$$

The algorithmic procedure for solving the problem is outlined next. The acceleration of block $\ddot{u}$ is calculated as follows:

1. If the root-sum-of-squares (RSS) of inertial forces due to movement of surface is greater than the Coulomb force, then the block will either start to slip or will keep sliding, but with a change in the slip direction:

1.1. If the previous step is sticking, then initiate slip motion and solve Eq. (6).

1.2. If both relative velocities are changing directions, then interpolate and solve using Eq. (6) to solve the interpolated points. Both sliding directions follow the direction of the surface accelerations.

1.3. If one relative velocity is changing direction, then interpolate and use Eq. (7) to solve the interpolated points. Only the direction of the changing relative velocity follows the direction of the surface acceleration.

1.4. Otherwise, the previous step is sliding, then keep sliding without change in directions and continue to solve Eq. (6).

2. If RSS of inertial forces due to the movement of the surface is less than the Coulomb force, and the previous step is sliding, then the block will keep sliding or start sticking from sliding:

2.1. If both relative velocities are changing directions, then cease sliding and start sticking. The acceleration of the block is the same as the acceleration of surface $(\ddot{u}(t)=\ddot{w}(t))$.

2.2. If one relative velocity is changing direction, then interpolate the time step and solve using Eq. (7). Only the direction of the changing relative velocity follows the direction of the surface acceleration.

2.3. Otherwise, keep sliding without change of direction and continue to solve Eq. (6).

3. If RSS of inertial forces due to movement of the surface is less than the Coulomb force, and the previous step is sticking, then keep sticking and the acceleration of the block is the same as the acceleration of surface.

\section{Validation}

For the purposes of validation, a two degree-of-freedom (2DOF) dynamic system is solved using the proposed and the traditional imaginary spring methods, focusing on transition points. The test parameters are $m=1 \mathrm{~kg}, \mu=0.3$ and $N_{o}=$ 9.81N. A time step of $0.2 \mathrm{~ms}$ is chosen. For the SMFM, a sticking stiffness of $40,000 \mathrm{~N} / \mathrm{m}$ is empirically selected. The problem is also solved in ABAQUS [6], to provide yet another solution to validate against. ABAQUS uses the SMFM method to handle the friction problem. The surface and block are discretized using rigid elements and the penalty contact method is used to define the interaction between the surface and block. 
As shown in Fig $2 \mathrm{a}$ and $2 \mathrm{~b}$, the velocity responses of the three models match well. However, accelerations, as shown in Fig 2c, 2d, 2g and $2 \mathrm{~h}$ exhibit significant differences. The ABAQUS model has considerable oscillations in the acceleration of the block. The acceleration response computed by the SMFM models also has oscillations at the transition points, but the results are better overall than the ABAQUS model. After a transition point, the acceleration and velocity responses exhibit oscillations because the spring force becomes a dominant factor in determining the motion of the block. As a result, SMFM has a delayed velocity response in comparison to the proposed model at transition points, as seen in Fig $2 \mathrm{e}$ and $2 \mathrm{f}$.

In contrast to the ABAQUS and SMFM model, the proposed model gives smooth and oscillation-free responses for acceleration, velocity. In addition, the response of proposed model shows that the response of the block rapidly follows the response of the surface after transition points are encountered. This indicates that the proposed model is able to capture the transition point properly and simulate responses in situations with near zero relative velocity.

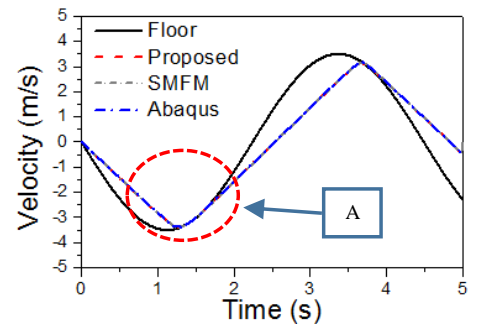

(a) Velocity $x$-direction

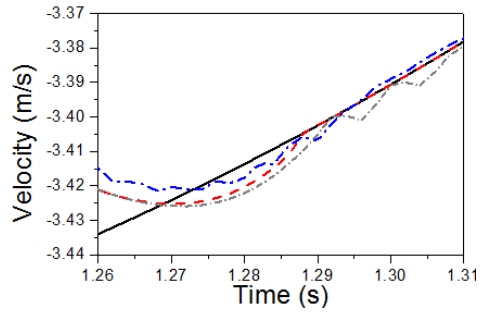

(e) Detail A

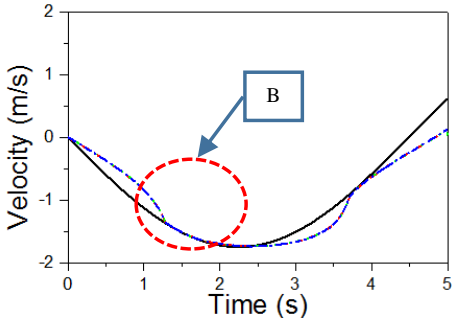

(b) Velocity $y$-direction

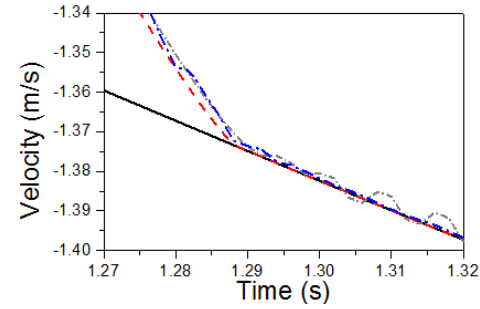

(f) Detail B

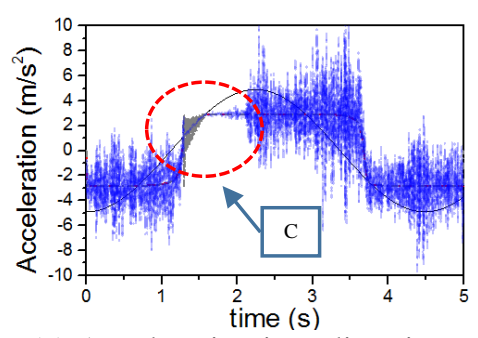

(c) Acceleration in $x$-direction

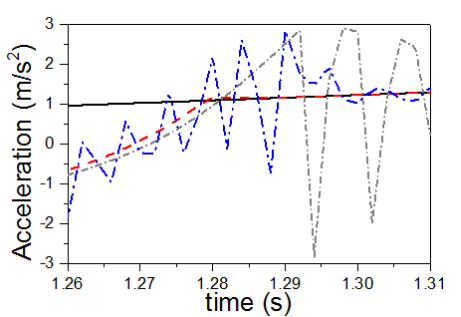

(g) Detail C

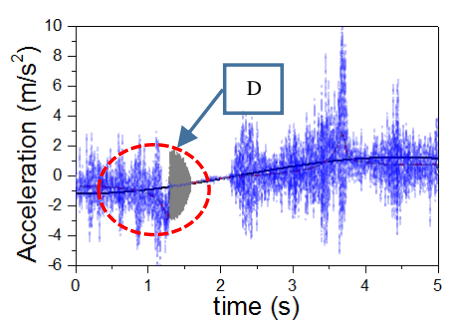

(d) Acceleration in $y$-direction

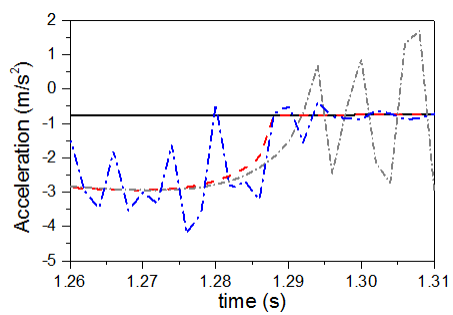

(h) Detail D

Fig 2. Typical dynamic response of friction models for two-dimensional motion.

\section{Conclusion}

A new model is proposed that addresses some key difficulties with previous stick-slip friction models. In particular, it resolves the problems that occur at near-zero relative velocity at the transition between slipping and sticking behavior. The model is validated by comparing its response to well-established models. It is shown that the model is able to capture velocity response as well as these other models, but without the accompanying severe oscillations in the acceleration response that plague other models.

\section{Acknowledgment}

The authors acknowledge the support of the National Science Foundation under Award Number 1362547.

\section{References}

[1] J. H. Griffin, "Friction damping of resonant stresses in gas turbine engine airfoils," Journal of Engineering for Power, vol. 102, no. 2, pp. 329-333, 1980.

[2] B. Westermo and F. Udwadia, "Periodic Response of a Sliding Oscillator System to Harmonic Excitation," Earthquake Engng. Struct. Dyn. Earthquake Engineering \& Structural Dynamics 11, no. 1, pp. 135-46, 1983.

[3] B. D. Yang and C. H. Menq, "Characterization of Contact Kinematics and Application to the design of Wedge Dampers in Turbomachinery Blading: part 1 - Stick-slip Contact Kinematics," ASME Journal of Engineering for Gas Turbine and Power, vol. 120, no. 2, 1998a.

[4] B. D. Yang and C. H. Menq, "Characterization of 3D contact kinematics and prediction of resonant response of structures having 3D frictional constraint," Journal of Sound and Vibration, vol. 217, no. 5, pp. 909-925, $1998 \mathrm{~b}$. 
[5] F. A. Tariku and R. J. Rogers, "Improved Dynamic Friction Models for Simulation of One-Dimensional and TwoDimensional Stick-Slip Motion," J. Tribol. Journal of Tribology, vol. 123, no. 4, pp. 661, 2001.

[6] (2011). Abaqus 6.11 Online Documentation. [Online]. 